\title{
A zero-free interval for chromatic polynomials of nearly 3-connected plane graphs*
}

\author{
F. M. Dong ${ }^{\dagger}$ \\ Mathematics and Mathematics Education \\ National Institute of Education \\ Nanyang Technical University, Singapore 637616 \\ e-mail: fengming.dong@nie.edu.sg \\ Bill Jackson \\ School of Mathematical Sciences \\ Queen Mary, University of London \\ Mile End Road, London E1 4NS, England \\ e-mail: b.jackson@qmul.ac.uk
}

March 23, 2010

\begin{abstract}
Let $G=(V, E)$ be a 2-connected plane graph on $n$ vertices with outer face $C$ such that every 2-vertex cut of $G$ contains at least one vertex of $C$. Let $P_{G}(q)$ denote the chromatic polynomial of $G$. We show that $(-1)^{n} P_{G}(q)>0$ for all $1<q \leq 1.2040 \ldots$. This result is a corollary of a more general result that $(-1)^{n} Z_{G}(q, \mathbf{w})>0$ for all $1<q \leq 1.2040 \ldots$, where $Z_{G}(q, \mathbf{w})$ is the multivariate Tutte polynomial of $G, \mathbf{w}=\left\{w_{e}\right\}_{e \in E}, w_{e}=-1$ for all $e$ which are not incident to a vertex of $C, w_{e} \in W_{2}$ for all $e \in E(C), w_{e} \in W_{1}$ for all other edges $e$, and $W_{1}, W_{2}$ are suitably chosen intervals with $-1 \in W_{1} \subset W_{2} \subseteq(-2,0)$.
\end{abstract}

Keywords: planar graph, Potts-model partition function, multivariate Tutte polynomial, chromatic polynomial, zeros

\section{Introduction}

The study of chromatic polynomials of graphs was initiated by Birkhoff [3] for planar graphs in 1912 and, for general graphs, by Whitney [14, 15] in 1932. Inspired by the 4-Colour Conjecture, Birkhoff and Lewis [4], obtained results concerning the distribution of the real zeros of chromatic polynomials of planar graphs and made the stronger conjecture that chromatic

\footnotetext{
* Supported by NIE AcRf funding (RI 5/06 DFM) of Singapore

${ }^{\dagger}$ Corresponding author
} 
polynomials of planar graphs have no real zeros greater than or equal to four. Their hope was that results from analysis and algebra could be used to prove their stronger conjecture and hence deduce that the 4-colour conjecture was true. This has not yet occured: indeed the 4-colour conjecture is now a theorem $[1,2,9]$, but the stronger conjecture of Birkhoff and Lewis remains unsolved. Nevertheless, many results have been obtained concerning the zero distribution of chromatic polynomials both on the real line and in the complex plane, see the survey articles $[6,10]$.

We will refer to the zeros of the chromatic polynomial $P_{G}(q)$ of a graph $G$ as chromatic roots of $G$. It follows from results of Tutte [13] and the second named author [5] that no graph can have a chromatic root in the real intervals $(-\infty, 0),(0,1)$ or $(1,32 / 27]$. Furthermore, these zero-free intervals are maximal: it is easy to see that 0 and 1 are chromatic roots of any graph with at least one edge, and a sequence of (planar) graphs with chromatic roots tending to $32 / 27$ from above is given in [5]. Thomassen [12] went further by showing that these are the only zero-free intervals for intervals for chromatic polynomials: for each $r \in(32 / 27, \infty)$ and all $\epsilon>0$ he constructed a graph which has a chromatic root in $(r-\epsilon, r+\epsilon)$.

The graphs used in the constructions of $[5,12]$ are 2-connected, but contain many 2-vertex cuts, so it is conceivable that the $(1,32 / 27]$ zero-free interval can be extended for the special family of 3-connected graphs. Indeed, it is conjectured [5, Conjecture $5(\mathrm{a})]$ that $(1, \alpha)$ is a zero-free interval for chromatic polynomials of 3 -connected graphs where $\alpha=1.781 \ldots$ is a chromatic root of $K_{3,4}$. For 3-connected planar graphs the smallest chromatic root we know is $\beta=1.840 \ldots$ which is a chromatic root of the Herschel graph. The main purpose of this paper is to give some evidence in support of the above mentioned conjecture. We will show:

Theorem 1.1 Let $G$ be a 2-connected plane graph on $n$ vertices and let $C$ be the bounding cycle of the outer face of $G$. Suppose that all 2-vertex cuts of $G$ contain at least one vertex of $C$. Then $(-1)^{n} P_{G}(q)>0$ for all $q \in(1, \gamma]$ where $\gamma=\left(5-\rho^{2}\right) / 4 \sim 1.2040 \ldots$ and $\rho \sim 0.4289 \ldots$ is a root of $x^{10}-8 x^{9}+12 x^{8}+40 x^{7}-82 x^{6}-72 x^{5}+68 x^{4}+56 x^{3}+313 x^{2}-16 x-56=0$.

We will also construct a sequence of graphs which satisfy the hypothesis of the theorem and have chromatic roots converging to $\gamma$ from above.

Our proof technique for Theorem 1.1 is inductive and uses a weighted generalization of the chromatic polynomial called the multivariate Tutte polynomial (or Pott's model partition function), see [11]. It is similar to that of a recent result of Jackson and Sokal [7] which extended the zero-free intervals $(-\infty, 0),(0,1)$ end $(1,32 / 27]$ from chromatic polynomials to multivariate Tutte polynomials.

An outline of the paper is as follows. Section 2 contains some preliminary graph theoretic results and introduces the multivariate Tutte polynomial. Our proof of (a weighted generalization of) Theorem 1.1 is given in Section 3. We construct the sequence of graphs which 
have chromatic roots converging to $\gamma$ in Section 4. We close by giving some open problems in Section 5.

\section{Definitions and preliminary results}

All graphs are allowed to have loops and multiple edges unless explicitly stated otherwise. We will refer to graphs with no loops or multiple edges as simple graphs. Let $G$ be a graph and $k$ be a non-negative integer. A $k$-separation of $G$ is a pair of subgraphs $\left\{G_{1}, G_{2}\right\}$ such that $G_{1} \cup G_{2}=G, E\left(G_{1}\right) \cap E\left(G_{2}\right)=\emptyset,\left|V\left(G_{1}\right) \cap V\left(G_{2}\right)\right|=k$, and $\left|E\left(G_{i}\right)\right| \geq k$ for each $i=1,2$. We refer to $V\left(G_{1}\right) \cap V\left(G_{2}\right)$ as a $k$-vertex-cut of $G$. The graph $G$ is $k$-connected if it has at least $k$ edges and has no $h$-separation for $0 \leq h<k$.

We shall need the following result which follows from a more general theorem of Mader [8, Satz 1].

Lemma 2.1 Let $G$ be a 2-connected simple graph and $C$ be a cycle in $G$. Suppose each vertex of $C$ has degree at least 3. Then $G-e$ is 2-connected for some e $\in E(C)$.

Let $G$ be a graph and $U \subseteq V(G)$. A bridge of $U$ in $G$ is a subgraph $B$ such that $B$ consists of either two vertices of $U$ joined by a single edge of $G$, or a component $H$ of $G-U$ together with the set of vertices $W \subseteq U$ which are adjacent to $H$ in $G$ and all edges of $G$ joining $H$ to $W$. We say that $B$ is a trivial bridge in the former case and is a non-trivial bridge in the latter case. We refer to the vertices of $U \cap V(B)$ as vertices of attachment of $B$ on $U$. When $F$ is a subgraph of $G$ we refer to the bridges of $V(F)$ in $G-E(F)$ as $F$-bridges in $G$.

\subsection{Nearly 3-connected plane graphs}

We use the term plane graph to mean a planar graph which has been imbedded in the plane. We assume that all minors of a plane graph $G$ are given an imbedding which has been obtained from that of $G$ by deleting and contracting edges. We say that $G$ is nearly 3-connected if it is 2-connected and, for all 2-separations $\left\{G_{1}, G_{2}\right\}$ of $G, V\left(G_{1}\right) \cap V\left(G_{2}\right)$ contains at least one vertex on the outer face of $G$. Our proof of Theorem 1.1 is inductive and will use the following hereditary properties of near 3-connectivity.

Lemma 2.2 Let $G$ be a nearly 3-connected plane graph and let $C$ be the bounding cycle of its outer face.

(a) Suppose that $e=u v \in E(C)$. Let $G^{\prime} \in\{G-e, G / e\}$. If $G^{\prime}$ is 2-connected then $G^{\prime}$ is nearly 3-connected. 
(b) Suppose that $\{F, H\}$ is a 2-separation of $G$ with $V(F) \cap V(H)=\{y, z\}$ and $C \subseteq H$. Let $F+h$ be obtained from $F$ by adding a new edge $h=y z$ in the outer face of $F$, and let $G^{\prime} \in\{F, F+h,(F+h) / h\}$. If $G^{\prime}$ is 2-connected then $G^{\prime}$ is nearly 3-connected.

Proof: We prove (a) and (b) simultaneously. Let $G^{\prime} \in\{G-e, G / e, F, F+h,(F+h) / h\}$ and assume that $G^{\prime}$ is 2-connected. Let $C^{\prime}$ be the bounding cycle of the outer face of $G^{\prime}$. Suppose $G^{\prime}$ has a 2-separation $\left\{G_{1}^{\prime}, G_{2}^{\prime}\right\}$ with $V\left(G_{1}^{\prime}\right) \cap V\left(G_{2}^{\prime}\right) \cap V\left(C^{\prime}\right)=\emptyset$. Relabeling if necessary, we have $V\left(C^{\prime}\right) \subseteq V\left(G_{2}^{\prime}\right)-V\left(G_{1}^{\prime}\right)$. Let $G_{2}=G-\left(V\left(G_{1}^{\prime}\right)-V\left(G_{2}^{\prime}\right)\right)-E\left(G_{1}^{\prime}\right)$. Then $\left\{G_{1}^{\prime}, G_{2}\right\}$ is a 2-separation of $G$ with $V(C) \subseteq V\left(G_{2}\right)-V\left(G_{1}^{\prime}\right)$. This contradicts the hypothesis that $G$ is nearly 3-connected.

\subsection{The multivariate Tutte polynomial}

Our proof of Theorem 1.1 will use the following extension of chromatic polynomials to weighted graphs. Let $G=(V, E)$ be a graph with vertex set $V$ and edge set $E$. The multivariate Tutte polynomial of $G$ is, by definition, the polynomial

$$
Z_{G}(q, \mathbf{w})=\sum_{A \subseteq E} q^{k(A)} \prod_{e \in A} w_{e}
$$

where $q$ and $\mathbf{w}=\left\{w_{e}\right\}_{e \in E}$ are commuting indeterminates, and $k(A)$ denotes the number of connected components in the subgraph $(V, A)$. We will refer to the pair $(G, \mathbf{w})$ as a weighted graph. We use $Z_{G}(q, w)$ to denote the two variable polynomial in which all edge weights are equal to $w$. This polynomial can be transformed to the standard Tutte polynomial by a simple change of variables, and satisfies $Z_{G}(q,-1)=P_{G}(q)$.

The following lemma gives a recursive procedure for calculating $Z_{G}(q, \mathbf{w})$.

Lemma 2.3 Let $(G, \mathbf{w})$ be a weighted graph and $e$ be an edge of $G$. Then $Z_{G}(q, \mathbf{w})=$ $Z_{G-e}\left(q,\left.\mathbf{w}\right|_{G-e}\right)+w_{e} Z_{G / e}\left(q,\left.\mathbf{w}\right|_{G-e}\right)$.

\section{Effective weights}

Suppose $(G, \mathbf{w})$ is a weighted graph and $F, H$ are connected subgraphs of $G$ with $F \cup H=G$, $E(F) \cap E(H)=\emptyset$ and $V(F) \cap V(H)=\{u, v\}$. We can calculate the 'effective weight' of $F$ in $(G, \mathbf{w})$ as follows. Let $\left(F+h,\left.\mathbf{w}\right|_{F},-1\right)$ be obtained from $\left(F,\left.\mathbf{w}\right|_{F}\right)$ by adding a new edge $h=u v$ to $F$ with weight -1 . Let $F_{u v}=(F+h) / h$. Let $\left(H+e_{F},\left.\mathbf{w}\right|_{H}, w_{F}\right)$ be obtained from $\left(H,\left.\mathbf{w}\right|_{H}\right)$ by adding an edge $e_{F}=u v$ with weight $w_{F}$. 
Lemma 2.4 Let

$$
w_{F}=\frac{q Z_{F_{u v}}\left(q,\left.\mathbf{w}\right|_{F}\right)-Z_{F}\left(q,\left.\mathbf{w}\right|_{F}\right)}{Z_{F}\left(q,\left.\mathbf{w}\right|_{F}\right)-Z_{F_{u v}}\left(q,\left.\mathbf{w}\right|_{F}\right)}
$$

Then

$$
Z_{G}(q, \mathbf{w})=\frac{Z_{F}\left(q,\left.\mathbf{w}\right|_{F}\right)-Z_{F_{u v}}\left(q,\left.\mathbf{w}\right|_{F}\right)}{q(q-1)} Z_{H+e_{F}}\left(q,\left.\mathbf{w}\right|_{H}, w_{F}\right)
$$

Furthermore, we have

$$
Z_{G}(q, \mathbf{w})=\frac{Z_{F+h}\left(q,\left.\mathbf{w}\right|_{F},-1\right)}{q(q-1)} Z_{H+e_{F}}\left(q,\left.\mathbf{w}\right|_{H}, w_{F}\right) .
$$

and

$$
\begin{aligned}
w_{F} & =\frac{(q-1) Z_{F_{u v}}\left(q,\left.\mathbf{w}\right|_{F}\right)}{Z_{F+h}\left(q,\left.\mathbf{w}\right|_{F},-1\right)}-1 \\
& =\frac{(q-1) Z_{F}\left(q,\left.\mathbf{w}\right|_{F}\right)}{Z_{F+h}\left(q,\left.\mathbf{w}\right|_{F},-1\right)}-q
\end{aligned}
$$

Proof: Equation (3) follows from [11, Proposition 4.2] (in particular equations (4.40) given in the proof of this proposition). Equations (4), (5) and (6) are simple reformulations of (2) using the fact that

$$
Z_{F+h}\left(q,\left.\mathbf{w}\right|_{F},-1\right)=Z_{F}\left(q,\left.\mathbf{w}\right|_{F}\right)-Z_{F_{u v}}\left(q,\left.\mathbf{w}\right|_{F}\right)
$$

We shall refer to the value of $w_{F}$ given in the above lemma as the effective weight of $F$ in $(G, \mathbf{w})$.

Two special cases of Lemma 2.4 are particularly useful: when $F$ is a cycle of length two (parallel reduction) and when $F$ is a path of length two (series reduction).

Lemma 2.5 Let $(G, \mathbf{w})$ be a weighted graph.

(a) Suppose $G$ has two edges $e_{1}, e_{2}$ with the same end vertices $u, v$. Let $H=G-\left\{e_{1}, e_{2}\right\}$ and let $\left(H+f,\left.\mathbf{w}\right|_{H}, w_{e_{1}} \| w_{e_{2}}\right)$ be obtained from $\left(H,\left.\mathbf{w}\right|_{H}\right)$ by adding a new edge $f=u v$ of weight

$$
w_{e_{1}} \| w_{e_{2}}=w_{e_{1}}+w_{e_{2}}+w_{e_{1}} w_{e_{2}} .
$$

Then $Z_{G}(q, \mathbf{w})=Z_{H+f}\left(q,\left.\mathbf{w}\right|_{H}, w_{e_{1}} \| w_{e_{2}}\right)$.

(b) Suppose $G$ has a vertex of degree two $w$ incident two edges $e_{1}=w u$ and $e_{2}=w v$. Let $H=G-w$ and let $\left(H+f,\left.\mathbf{w}\right|_{H}, w_{e_{1}} \bowtie_{q} w_{e_{2}}\right)$ be obtained from $\left(H,\left.\mathbf{w}\right|_{H}\right)$ by adding a new edge $f=u v$ of weight

$$
w_{e_{1}} \bowtie_{q} w_{e_{2}}=\frac{w_{e_{1}} w_{e_{2}}}{q+w_{e_{1}}+w_{e_{2}}} .
$$

Then $Z_{G}(q, \mathbf{w})=\left(q+w_{e_{1}}+w_{e_{2}}\right) Z_{H+f}\left(q,\left.\mathbf{w}\right|_{H}, w_{e_{1}} \bowtie_{q} w_{e_{2}}\right)$. 


\section{$3 \quad$ Main Result}

Our proof of Theorem 1.1 is inductive and is based on using local operations, such as parallel and series reduction of edges incident to vertices on $C$, to transform $G$ to a smaller nearly 3connected plane graph. This requires us to consider weighted edges: we use the multivariate Tutte polynomial $Z_{G}(q, \mathbf{w})$ where $q \in(1, \gamma], w_{e}=-1$ when $e$ is not incident to a vertex of $C, w_{e} \in W_{1}(q)$ when $e$ is incident to a vertex of $C$ but $e \notin E(C)$, and $w_{e} \in W_{2}(q)$ when $e \in E(C)$, for suitably chosen intervals $W_{1}(q), W_{2}(q) \subseteq(-2,0)$ which we will define below.

\section{The interval $\mathbf{W}_{1}$}

We take $W_{1}=\left(a_{1}, b_{1}\right)$ where $a_{1}=(-1) \bowtie_{q} b_{1}$ and $b_{1}$ is the smallest real root of the equation $w=\left[(-1) \bowtie_{q} w\right] \|\left[(-1) \bowtie_{q} w\right]$. This gives $-w\left(w^{2}+w(2 q-1)+q^{2}-1\right)(q-1+w)^{-2}=0$ and hence

$$
b_{1}=-q+\frac{1}{2}-\frac{1}{2} \sqrt{5-4 q}
$$

and

$$
a_{1}=\frac{-3+\sqrt{5-4 q}}{2} .
$$

The interval $W_{1}$ is defined for all $q \in(1,1.25]$.

\section{The interval $\mathbf{W}_{2}$}

We take $W_{2}=\left(a_{2}, b_{2}\right)$ where $a_{2}=b_{2} \bowtie_{q} b_{2}, b_{2}=\min \left\{b_{2,1}, b_{2,2}\right\}$, and $b_{2,1}, b_{2,2}$ are defined as follows. We let $b_{2,1}$ be the unique real root of the equation $Z_{K_{3}}(q, w, w, w)=0$. We have $Z_{K_{3}}(q, w, w, w)=q\left(w^{3}+3 w^{2}+3 q w+q^{2}\right)$ and hence

$$
b_{2,1}=-1+(q-1)^{1 / 3}-(q-1)^{2 / 3}=-\frac{q}{1+(q-1)^{1 / 3}} .
$$

We let $b_{2,2}$ be the largest real root of the equation $w=\left[w \bowtie_{q} w\right] \|\left[b_{1} \bowtie_{q} b_{1}\right]$. We have $w-\left(\left[w \bowtie_{q} w\right] \|\left[b_{1} \bowtie_{q} b_{1}\right]\right)=g(w)\left(q+2 b_{1}\right)^{-1}(q+2 w)^{-1}$ where

$$
g(w)=\left(b_{1}^{2}-2 b_{1}-q\right) w^{2}+\left(2 b_{1}^{2}-2 b_{1} q-q^{2}\right) w+b_{1}^{2} q .
$$

It can be seen that $g(w)=0$ has real roots if and only if $q \leq \gamma$. It can also be seen that the two curves $b_{2,1}$ and $b_{2,2}$ have a unique point of intersection which occurs when $g\left(b_{2,1}\right)=0$. Solving for $q$ we obtain $q=\tau=1+\theta^{3} \sim 1.1956 \ldots$, where $\theta \sim 0.5805 \ldots$ is a root of $x^{14}-2 x^{12}+6 x^{11}-x^{10}-10 x^{9}+15 x^{8}-6 x^{7}-10 x^{6}+15 x^{5}-12 x^{4}+7 x^{3}-4 x^{2}+3 x-1=0$. We have $b_{2,1}<b_{2,2}$ for $1<q<\tau$ and $b_{2,1}>b_{2,2}$ for $\tau<q \leq \gamma$. Thus

$$
b_{2}= \begin{cases}b_{2,1} & \text { for } q \in(1, \tau) \\ b_{2,2} & \text { for } q \in[\tau, \gamma]\end{cases}
$$




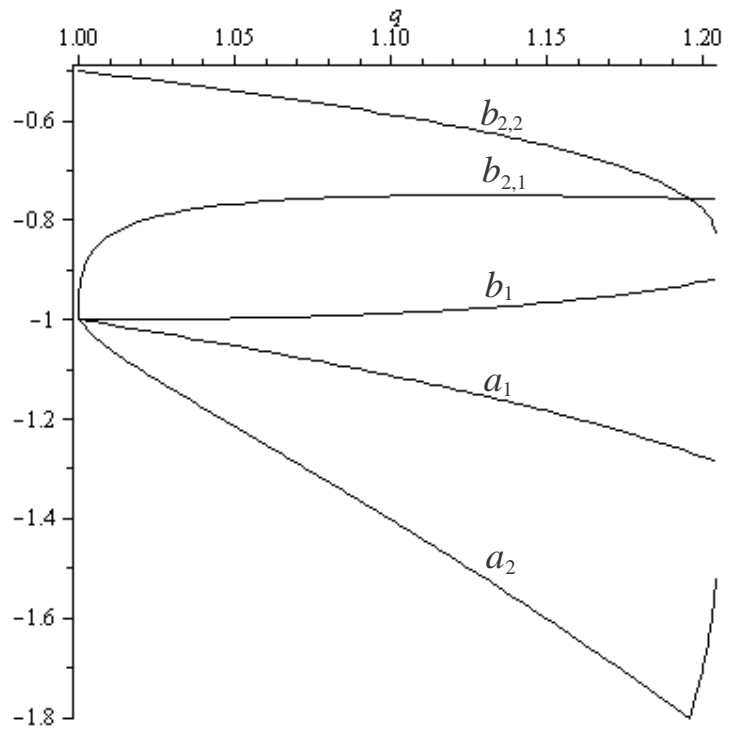

Figure 1: The intervals $W_{1}=\left(a_{1}, b_{1}\right)$ and $W_{2}=\left(a_{2}, b_{2}\right)$ for $1<q \leq 1.204 \cdots$.

The interval $W_{2}$ is defined for all $q \in(1, \gamma]$.

We will use the intervals $W_{1}$ and $W_{2}$ to prove the following weighted generalisation of Theorem 1.1 .

Theorem 3.1 Let $(G, \mathbf{w})$ be a weighted nearly 3 -connected plane graph on $n$ vertices and let $C$ be the bounding cycle of its outer face. Let $q \in(1, \gamma]$, where $\gamma=\left(5-\rho^{2}\right) / 4 \sim 1.2040 \ldots$ and $\rho \sim 0.4289 \ldots$ is a root of $x^{10}-8 x^{9}+12 x^{8}+40 x^{7}-82 x^{6}-72 x^{5}+68 x^{4}+56 x^{3}+313 x^{2}-16 x-56=$ 0. Suppose that:

(a) $w_{e}=-1$ for all $e \in E(G-C)$;

(b) $w_{e} \in W_{1}$ for all $e \in E(G)-E(C)-E(G-C)$;

(c) $w_{e} \in W_{2}$ for all $e \in E(C)$.

Then $(-1)^{n} Z_{G}(q, \mathbf{w})>0$.

We first need to establish some properties of the intervals $W_{1}, W_{2}$. We will use the following result which can be verified by elementary calculus.

Lemma 3.2 Let $z_{1}, q$ be fixed real numbers with $q>0$. 
(a) For all $z \in(-\infty, \infty)$ : $z_{1} \| z=-1$ when $z_{1}=-1$, $z_{1} \| z$ is a strictly increasing continuous function of $z$ when $z_{1}>-1$, and $z_{1} \| z$ is a strictly decreasing continuous function of $z$ when $z_{1}<-1$.

(b) For all $z \in\left(-\infty,-q-z_{1}\right) \cup\left(-q-z_{1}, \infty\right)$ : $z_{1} \bowtie_{q} z=-q$ when $z_{1}=-q, z_{1} \bowtie_{q} z$ is a strictly increasing continuous function of $z$ when $z_{1} \in(-\infty,-q) \cup(0, \infty)$, and $z_{1} \bowtie_{q} z$ is a strictly decreasing continuous function of $z$ when $z_{1} \in(-q, 0)$.

Given $q \in \mathbb{R}$ and $X_{1}, X_{2} \subset \mathbb{R}$ we put $X_{1} \| X_{2}=\left\{x_{1} \| x_{2}: x_{1} \in X_{1}, x_{2} \in X_{2}\right\}$ and $X_{1} \bowtie_{q} X_{2}=\left\{x_{1} \bowtie_{q} x_{2}: x_{1} \in X_{1}, x_{2} \in X_{2}\right\}$. We may use Lemma 3.2 to determine what happens when these operations are applied to intervals.

Lemma 3.3 Let $q, c_{1}, d_{1}, c_{2}, d_{2} \in \mathbb{R}$ and put $X_{1}=\left(c_{1}, d_{1}\right)$ and $X_{2}=\left(c_{2}, d_{2}\right)$. (a) If $c_{1}, c_{2}<$ -1 and $d_{1}, d_{2}>-1$ then

$$
X_{1} \| X_{2}=\left(\min \left\{c_{1}\left\|d_{2}, d_{1}\right\| c_{2}\right\}, \max \left\{c_{1}\left\|c_{2}, d_{1}\right\| d_{2}\right\}\right)
$$

(b) If $c_{1}, c_{2}<-q$ and $-q<d_{1}, d_{2}<-q / 2$ then

$$
X_{1} \bowtie_{q} X_{2}=\left(\min \left\{c_{1} \bowtie_{q} c_{2}, d_{1} \bowtie_{q} d_{2}\right\}, \max \left\{c_{1} \bowtie_{q} d_{2}, d_{1} \bowtie_{q} c_{2}\right\}\right)
$$

We can now obtain our desired results for $W_{1}, W_{2}$.

Lemma 3.4 Let $q \in(1,1.25]$. Then:

(a) $a_{1}<-q<-1<b_{1}$;

(b) $W_{1} \| W_{1} \subseteq W_{1}$;

(c) $W_{1} \bowtie_{q}[-1] \subseteq W_{1}$.

Proof: (a) This follows directly from the definitions of $a_{1}$ and $b_{1}$ on page 6 .

(b) By (a), we have $a_{1}<-1<b_{1}$. Lemma 3.3(a) implies that $W_{1} \| W_{1}=\left(a_{1} \| b_{1}\right.$, $\max \left\{a_{1} \|\right.$ $\left.\left.a_{1}, b_{1} \| b_{1}\right\}\right)$. We have $\left(a_{1} \| b_{1}\right)-a_{1}=\left(a_{1}+1\right) b_{1}>0$, so $a_{1} \| b_{1}>a_{1}$. Similarly $\left(b_{1} \| b_{1}\right)-b_{1}=b_{1}\left(b_{1}+1\right)<0$ so $b_{1} \| b_{1}<b_{1}$. We also have

$$
a_{1}\left\|a_{1}=\left([-1] \bowtie_{q} b_{1}\right)\right\|\left([-1] \bowtie_{q} b_{1}\right)=b_{1}
$$

by the definition of $W_{1}$. Thus $W_{1} \| W_{1}=\left(a_{1} \| b_{1}, b_{1}\right) \subseteq W_{1}$.

(c) Since $-1 \in(-q, 0)$, Lemma 3.3(b) implies that

$$
W_{1} \bowtie_{q}[-1]=\left(b_{1} \bowtie_{q}[-1], a_{1} \bowtie_{q}[-1]\right)=\left(a_{1}, a_{1} \bowtie_{q}[-1]\right) .
$$


Furthermore

$$
a_{1} \bowtie_{q}[-1]-b_{1}=\frac{2(q-2)(q-2+\sqrt{5-4 q})}{(2 q-5+\sqrt{5-4 q})}<0
$$

for $1<q \leq 1.25$. Thus $a_{1} \bowtie_{q}[-1]<b_{1}$ and so $W_{1} \bowtie_{q}[-1] \subseteq W_{1}$.

Lemma 3.5 Let $q \in(1, \gamma]$. Then:

(a) $a_{2}<a_{1}<-q<-1<b_{1}<b_{2}<-q / 2$;

(b) $W_{1} \subseteq W_{2}$;

(c) $W_{2} \bowtie_{q} W_{2} \subseteq W_{2}$;

(d) $\left(W_{1} \bowtie_{q} W_{1}\right) \| W_{2} \subseteq W_{2}$;

(e) $W_{1} \| W_{2} \subseteq W_{2}$.

Proof: (a) By Lemma 3.4(a), it suffices to show that $a_{2}<a_{1}$ and $b_{1}<b_{2}<-q / 2$. We first show that

$$
b_{1}<b_{2}<-q / 2
$$

We consider the cases when $q \leq \tau$ and $q \geq \tau$ separately.

Case 1: $1<q \leq \tau$. We have $b_{2}+\frac{q}{2}=-\frac{q}{1+(q-1)^{1 / 3}}+\frac{q}{2}<0$, for all $1<q<2$ so $b_{2}<-q / 2$. Furthermore,

$$
\begin{aligned}
b_{2}-b_{1} & =\frac{-q}{1+(q-1)^{1 / 3}}+q-\frac{1}{2}+\frac{1}{2} \sqrt{5-4 q} \\
& =\frac{(q-1)^{1 / 3}\left(q \sqrt{5-4 q}+2-q-2(q-1)^{1 / 3}\right)}{\left(1+(q-1)^{1 / 3}\right)(1+\sqrt{5-4 q})} .
\end{aligned}
$$

Since $2-q-2(q-1)^{1 / 3}>0$ for $1 \leq q \leq 1.23$, we have $b_{2}>b_{1}$.

Case 2: $\tau<q \leq \gamma$. We have $b_{2}=b_{2,2}$ where $b_{2,2}$ is the largest root of $g(x)=0$ and

$$
g(x)=\left(b_{1}^{2}-2 b_{1}-q\right) x^{2}+\left(2 b_{1}^{2}-2 b_{1} q-q^{2}\right) x+b_{1}^{2} q .
$$

Let $b_{2,2}^{-}$be the other root. Then $b_{2,2}^{-} \leq b_{2,2}$ and

$$
\frac{b_{2,2}^{-}+b_{2,2}}{2}=-\frac{2 b_{1}^{2}-2 b_{1} q-q^{2}}{2\left(b_{1}^{2}-2 b_{1}-q\right)} .
$$

We have $2 b_{1}+q=-q+1-\sqrt{5-4 q}<0$ for $1<q<1.25$. Thus

$$
b_{1}^{2}-2 b_{1}-q>0
$$

Hence the coefficient of $x^{2}$ in $g(x)$ is positive and we have $g(x) \leq 0$ for $b_{2,2}^{-} \leq x \leq b_{2,2}$. Thus, to show that $b_{2,2}<-q / 2$, it will suffice to show that $\left(b_{2,2}^{-}+b_{2,2}\right) / 2<-q / 2$ and $g(-q / 2)>0$. We have

$$
\frac{b_{2,2}^{-}+b_{2,2}}{2}+\frac{q}{2}=-\frac{2 b_{1}^{2}-2 b_{1} q-q^{2}}{2\left(b_{1}^{2}-2 b_{1}-q\right)}+\frac{q}{2}=\frac{b_{1}^{2}(2-q)}{2\left(q-b_{1}^{2}+2 b_{1}\right)}<0
$$


by (8), and

$$
g(-q / 2)=q^{2}\left(q+2 b_{1}+b_{1}^{2}\right) / 4=q^{2}\left(q-1+\left(b_{1}+1\right)^{2}\right) / 4>0 .
$$

Hence $b_{2,2}<-q / 2$.

It remains to show that $b_{2,2}>b_{1}$. We have

$$
\frac{b_{2,2}^{-}+b_{2,2}}{2}-b_{1}=\frac{q^{2}-6 b_{1}^{2}+2 b_{1}^{3}}{2\left(q-b_{1}^{2}+2 b_{1}\right)} .
$$

Furthermore

$$
q^{2}-6 b_{1}^{2}+2 b_{1}^{3}=-\left(5-4 q^{2}+2 q^{3}\right)-\left(3 q^{2}-1+2 q\right) \sqrt{5-4 q} .
$$

Since $5-4 q^{2}+2 q^{3}>0$ and $3 q^{2}-1+2 q>0$ for $q \in(1,1.25)$ we have $q^{2}-6 b_{1}^{2}+2 b_{1}^{3}<0$. We may now use (8) and (9) to deduce that $\left(b_{2,2}^{-}+b_{2,2}\right) / 2-b_{1}>0$ and hence $b_{2,2} \geq\left(b_{2,2}^{-}+b_{2,2}\right) / 2>b_{1}$.

This completes the proof of (7). Using (7), Lemma 3.2(b), and the definitions of $a_{1}, a_{2}$, we have

$$
a_{2}=b_{2} \bowtie_{q} b_{2}<(-1) \bowtie_{q} b_{1}=a_{1}
$$

(b) This follows immediately from (a).

(c) Since $a_{2}<-q<b_{2}$ by (a), Lemma 3.3(b) implies that

$$
W_{2} \bowtie_{q} W_{2}=\left(\min \left\{a_{2} \bowtie_{q} a_{2}, b_{2} \bowtie_{q} b_{2}\right\}, a_{2} \bowtie_{q} b_{2}\right) .
$$

We have $b_{2} \bowtie_{q} b_{2}=a_{2}$ by the definition of $a_{2}$. Furthermore (a) implies that $\left(a_{2} \bowtie_{q} a_{2}\right)-a_{2}=$ $-a_{2}\left(a_{2}+q\right) /\left(q+2 a_{2}\right)>0$ and $\left(a_{2} \bowtie_{q} b_{2}\right)-b_{2}=-b_{2}\left(b_{2}+q\right) /\left(q+a_{2}+b_{2}\right)<0$. Thus $W_{2} \bowtie_{q} W_{2}=\left(a_{2}, a_{2} \bowtie_{q} b_{2}\right) \subseteq W_{2}$.

(d) Since $a_{1}<-q<b_{1}$, Lemma 3.3(b) implies that

$$
W_{1} \bowtie_{q} W_{1}=\left(\min \left\{a_{1} \bowtie_{q} a_{1}, b_{1} \bowtie_{q} b_{1}\right\}, a_{1} \bowtie_{q} b_{1}\right) .
$$

We have

$$
\left(b_{1} \bowtie_{q} b_{1}\right)-\left(a_{1} \bowtie_{q} a_{1}\right)=\frac{(2-q)(\sqrt{5-4 q}-1)^{2}(\sqrt{5-4 q}-3)^{2}}{16(q-1+\sqrt{5-4 q})(q-3+\sqrt{5-4 q})}<0
$$

for $1<q \leq 1.25$. Hence $b_{1} \bowtie_{q} b_{1}<a_{1} \bowtie_{q} a_{1}$. We also have $\left(a_{1} \bowtie_{q} b_{1}\right)-b_{1}=-b_{1}\left(q+b_{1}\right)(q+$ $\left.a_{1}+b_{1}\right)^{-1}<0$ by (a). Thus $a_{1} \bowtie_{q} b_{1}<b_{1}$. Hence

$$
W_{1} \bowtie_{q} W_{1} \subseteq\left(b_{1} \bowtie_{q} b_{1}, b_{1}\right) .
$$

Since $b_{1} \in(-q, 0)$, Lemma 3.2(b) implies that $z \bowtie_{q} b_{1}$ is a strictly decreasing function of $z$. Hence $b_{1} \bowtie_{q} b_{1}<(-q) \bowtie_{q} b_{1}=-q<-1$. Since we also have $a_{1}<-1<b_{1}<b_{2}$ we can use Lemma 3.3(a) and (10) to deduce that

$$
\left(W_{1} \bowtie_{q} W_{1}\right) \| W_{2} \subseteq\left(\min \left\{\left(b_{1} \bowtie_{q} b_{1}\right)\left\|b_{2}, b_{1}\right\| a_{2}\right\}, \max \left\{\left(b_{1} \bowtie_{q} b_{1}\right)\left\|a_{2}, b_{1}\right\| b_{2}\right\}\right)
$$


We have $\left(b_{1} \| a_{2}\right)-a_{2}=b_{1}\left(a_{2}+1\right)>0$ and hence

$$
b_{1} \| a_{2}>a_{2}
$$

Similarly $\left(b_{1} \| b_{2}\right)-b_{2}=b_{1}\left(b_{2}+1\right)<0$ so

$$
b_{1} \| b_{2}<b_{2}
$$

We also have

$$
\begin{aligned}
& {\left[\left(b_{1} \bowtie_{q} b_{1}\right) \| b_{2}\right]-a_{2}} \\
& \quad=\frac{b_{1}^{2} b_{2}}{q+2 b_{1}}+\frac{b_{1}^{2}}{q+2 b_{1}}+b_{2}-\frac{b_{2}^{2}}{q+2 b_{2}} \\
& \quad=\frac{b_{2}\left(q+2 b_{1}\right)\left([q-1]+\left[b_{1}+1\right]\left[b_{2}+1\right]\right)+b_{1} q\left(1+b_{2}\right)\left(b_{1}-b_{2}\right)}{\left(q+2 b_{1}\right)\left(q+2 b_{2}\right)} \\
& \quad>0,
\end{aligned}
$$

as both the numerator and denominator are positive. Thus $\left(b_{1} \bowtie_{q} b_{2}\right) \| b_{2}>a_{2}$.

It remains to show that $\left(b_{1} \bowtie_{q} b_{1}\right) \| a_{2} \leq b_{2}$. From the definition of $W_{2}$, we have $a_{2}=b_{2} \bowtie_{q} b_{2}$ and

$$
b_{2}-\left[\left(b_{1} \bowtie_{q} b_{1}\right) \|\left(b_{2} \bowtie_{q} b_{2}\right)\right]=g\left(b_{2}\right)\left(q+2 b_{1}\right)^{-1}\left(q+2 b_{2}\right)^{-1} .
$$

When $q \in[\tau, \gamma]$ we have $b_{2}=b_{2,2}, g\left(b_{2,2}\right)=0$ and hence $\left(b_{1} \bowtie_{q} b_{1}\right) \| a_{2}=b_{2}$. When $q \in(1, \tau)$ we have $b_{2}=b_{2,1}$. Since $\tau$ is the unique root of $g\left(b_{2,1}\right)=0, g\left(b_{2,1}\right)$ has constant sign for $q \in(1, \tau)$. It is not difficult to check that this sign is positive. Thus $b_{2}-\left[\left(b_{1} \bowtie_{q} b_{1}\right) \| a_{2}=\right.$ $g\left(b_{2}\right)\left(q+2 b_{1}\right)^{-1}\left(q+2 b_{2}\right)^{-1}>0$. So in both cases we have

$$
\left(b_{1} \bowtie_{q} b_{1}\right) \| a_{2} \leq b_{2} .
$$

(e) Since $a_{2}<a_{1}<-q<-1<b_{1}<b_{2}$ by (a), Lemma 3.3(a) implies that

$$
W_{1} \| W_{2}=\left(\min \left\{a_{1}\left\|b_{2}, b_{1}\right\| a_{2}\right\}, \max \left\{a_{1}\left\|a_{2}, b_{1}\right\| b_{2}\right\}\right)
$$

We have $b_{1} \| a_{2}>a_{2}$ and $b_{1} \| b_{2}<b_{2}$ by (11) and (12), respectively. We also have $\left(a_{1} \| b_{2}\right)-a_{1}=b_{2}\left(a_{1}+1\right)>0$. Hence $a_{1} \| b_{2}>a_{1}>a_{2}$. It remains to show that $a_{1} \| a_{2} \leq b_{2}$.

Since $b_{1} \in(-q, 0)$, Lemma 3.2(b) implies that $z \bowtie_{q} b_{1}$ is a strictly decreasing function of $z$ and hence $a_{1}=(-1) \bowtie_{q} b_{1}>b_{1} \bowtie_{q} b_{1}$. Since $a_{2}<-1$, Lemma 3.2(a) implies that $z \| a_{2}$ is also a strictly decreasing function of $z$ and hence

$$
a_{1}\left\|a_{2}<\left(b_{1} \bowtie_{q} b_{1}\right)\right\| a_{2} \leq b_{2}
$$

by (13). 


\section{Proof of Theorem 3.1}

Suppose the theorem is false and choose a counterexample $(G, w)$ with as few edges as possible. We can use the parallel reduction formula Lemma 2.5(a), and the facts that $W_{1} \| W_{1} \subseteq W_{1}$ and $W_{1} \| W_{2} \subseteq W_{2}$ by Lemmas 3.4(b) and 3.5(e), to deduce that no edge of $G$ is parallel to an edge in $E(G)-E(C)$. (We do not have $W_{2} \| W_{2} \subseteq W_{2}$ so we cannot deduce that $|E(C)| \neq 2$.)

Claim 3.6 $G \neq K_{2}^{(2)}$.

Proof: Suppose $G=K_{2}^{(2)}$. Then $Z_{G}\left(q, w_{1}, w_{2}\right)=q\left(q+w_{1}+w_{2}+w_{1} w_{2}\right)$ so we need to show that $f\left(w_{1}, w_{2}\right)=q+w_{1}+w_{2}+w_{1} w_{2}>0$ for $w_{1}, w_{2} \in W_{2}$. Recall that $b_{2,1}=$ $-1+(q-1)^{1 / 3}-(q-1)^{2 / 3}$. Let $a_{2,1}=b_{2,1} \bowtie_{q} b_{2,1}$. Since $-q<b_{1} \leq b_{2,1}$, Lemma 3.2(b) implies that $a_{2,1} \leq a_{2}$ and hence $W_{2} \subseteq\left(a_{2,1}, b_{2,1}\right)$. We will consider the extreme values of $f$ for $w_{1}, w_{2} \in\left[a_{2,1}, b_{2,1}\right]$. Since $\frac{\delta f}{\delta w_{1}}=1+w_{2}, f$ changes monotonically with $w_{1}$ when $w_{2}$ is fixed. It follows that we need only consider what happens when $w_{1} \in\left\{a_{2,1}, b_{2,1}\right\}$, and similarly $w_{2} \in\left\{a_{2,1}, b_{2,1}\right\}$. We have $f(w, w)=q+(1+w)^{2}-1>0$ since $q>1$. Thus we need only consider $f\left(a_{2,1}, b_{2,1}\right)$. Since $a_{2,1}=b_{2,1} \bowtie_{q} b_{2,1}$, we have

$$
q f\left(a_{2,1}, b_{2,1}\right)=Z_{G}\left(a_{2,1}, b_{2,1}\right)=\frac{1}{q+2 b_{2,1}} Z_{K_{3}}\left(b_{2,1}, b_{2,1}, b_{2,1}\right)=0
$$

by the definition of $b_{2,1}$. Since $f\left(w_{1}, w_{2}\right)$ is strictly increasing with $w_{1}$ and strictly decreasing with $w_{2}$ when the point $\left(w_{1}, w_{2}\right)$ is close to the point $\left(a_{2,1}, b_{2,1}\right)$ we may deduce that $f\left(w_{1}, w_{2}\right)>0$ for all $w_{1}, w_{2}$ in the open interval $\left(a_{2,1}, b_{2,1}\right)$.

Claim 3.7 Let $F, H$ be connected subgraphs of $G$ with $G=F \cup H, E(F) \cap E(H)=\emptyset$, $V(F) \cap V(H)=\{u, x\}$, and $C \subseteq H$. Suppose that $u \in V(C)$ and that $F$ is 2-connected if it has at least two edges. Let $F+h$ be obtained by adding a new edge $h=u x$ to $F$ and put $F_{u x}=(F+h) / h$. Suppose that either $F_{u x}$ is 2-connected or $x \notin V(C)$. Then the effective weight $w_{F}$ of $F$ in $(G, \mathbf{w})$ is contained in $W_{1}$.

Proof: We use an inner induction on $|E(F)|$. If $E(F)=\{e\}$ then $w_{F}=w_{e} \in W_{1}$ by an hypothesis of the theorem. Hence we may suppose that $|E(F)| \geq 2$ and thus $F$ is 2-connected. Let $L_{1}, L_{2}, \ldots, L_{t}$ be the $u x$-bridges in $F$. Since $W_{1} \| W_{1} \subseteq W_{1}$ by Lemma 3.4(b), it will suffice to show that the effective weight $w_{L_{i}}$ of $L_{i}$ in $(G, \mathbf{w})$ is contained in $W_{1}$ for all $1 \leq i \leq t$. This is clearly true if $\left|E\left(L_{i}\right)\right|=1$ and hence we may assume that $\left|E\left(L_{i}\right)\right| \geq 2$.

Suppose $t=1$. Then $L_{1}=F$. By hypothesis $F$ and $F+h$ are both 2-connected, the fact that $t=1$ implies that $F_{u x}$ is also 2-connected. Lemma 2.2(b) now implies that $F, F+h$ 
and $F_{u x}$ are all nearly 3 -connected. By Lemma 2.4 , we have

$$
\begin{aligned}
w_{F} & =\frac{(q-1) Z_{F}\left(q,\left.\mathbf{w}\right|_{F}\right)}{Z_{F+h}\left(q,\left.\mathbf{w}\right|_{F},-1\right)}-q \\
& =\frac{(q-1) Z_{F_{u x}}\left(q,\left.\mathbf{w}\right|_{F}\right)}{Z_{F+h}\left(q,\left.\mathbf{w}\right|_{F-e},-1\right)}-1 .
\end{aligned}
$$

By the outer induction $Z_{F}\left(q,\left.\mathbf{w}\right|_{F}\right) / Z_{F+h}\left(q,\left.\mathbf{w}\right|_{F},-1\right)>0$ and hence $w_{F}>-q$ by (14). Similary $Z_{F_{u x}}\left(q,\left.\mathbf{w}\right|_{F}\right) / Z_{F+h}\left(q,\left.\mathbf{w}\right|_{F},-1\right)<0$ and hence $w_{F}<-1$ by (15). Thus $w_{F} \in W_{1}$.

Hence we may assume that $t \geq 2$. This implies that $F_{u v}$ is not 2-connected and hence, by hypothesis, $x \notin V(C)$. It also imples that $L_{i}$ has fewer edges than $F$. Hence, if $L_{i}$ is 2 connected, then we have $w_{L_{i}} \in W_{1}$ by the inner induction. Thus we may assume that $L_{i}$ is not 2-connected. The hypotheses that all 2-vertex-cuts of $G$ intersect $C$ and that $x \notin V(C)$ now imply that $L_{i}$ has exactly two blocks, $B_{1}, B_{2}$, with $u \in V\left(B_{1}\right), x \in V\left(B_{2}\right)$ and $\left|V\left(B_{2}\right)\right|=2$. Then the effective weight $w_{B_{1}} \in W_{1}$ by the inner induction, and $w_{B_{2}}=-1$ since $\left|E\left(B_{2}\right)\right|=1$ and $B_{2}$ is not incident with a vertex of $C$. Thus

$$
w_{L_{i}}=w_{B_{1}} \bowtie_{q}[-1] \in W_{1} \bowtie_{q}[-1] \subseteq W_{1}
$$

by Lemma 3.4(c).

Claim 3.8 Each vertex of $C$ has degree at least three.

Proof: Suppose $C$ contains a vertex $y$ of degree two and let $e_{1}=y u, e_{2}=y v$ be the edges incident with $y$. Then $e_{1}, e_{2} \in E(C)$. Let $H=G-y$ and let $H+f$ be obtained from $H$ by adding a new edge $f=u v$ in the outer face of $H$. Then the bounding cycle of the outer face of $H+f$ is $C^{\prime}=C-y+f$. It is not difficult to see that $H+f$ is 2-connected and we may now use Lemma 2.2(a) to deduce that $H+f$ is nearly 3 -connected (we have $H+f=G / e_{2}$ ). By Lemma $2.5(\mathrm{~b})$ we have $Z_{G}(q, \mathbf{w})=\left(q+w_{e_{1}}+w_{e_{2}}\right) Z_{H+f}\left(q,\left.\mathbf{w}\right|_{H}, w_{e_{1}} \bowtie_{q} w_{e_{2}}\right)$. Since $w_{e_{1}}, w_{e_{2}} \in W_{2}$, Lemma 3.5(a) implies that $q+w_{e_{1}}+w_{e_{2}}<0$ and Lemma 3.5(c) gives $w_{e_{1}} \bowtie_{q} w_{e_{2}} \in W_{2}$. We may now use induction to deduce that $(-1)^{n} Z_{G}(q, \mathbf{w})>0$.

If $|V(C)|=2$ then $G-e$ is 2-connected for each edge $e=u v \in E(C)$. On the other hand, if $|V(C)| \geq 3$, then $G$ is simple and, by Claim 3.8 and Lemma 2.1, we may choose $e=u v \in E(C)$ such that $G-e$ is 2-connected. We may now use Lemma 2.2(a) to deduce that $G-e$ is nearly 3 -connected. If $G / e$ were 2 -connected then Lemma 2.2(a) would imply that $G / e$ is nearly 3 -connected, and we could use Lemma 2.3 and induction to deduce that $(-1)^{n} Z_{G}(q, \mathbf{w})>0$. Hence $G / e$ is not 2-connected.

Let $C^{*}$ be the face of $G$ which contains $e$ and is distinct from $C$. As noted at the beginning of the proof, no edge in $E(G)-E(C)$ can be parallel to $e$. Hence $\left|V\left(C^{*}\right)\right| \geq 3$. Let $B$ be 
the $C$-bridge of $G$ which contains $V\left(C^{*}\right)-\{u, v\}$. Since $G / e$ is not 2-connected the set of attachments of $B$ on $C$ is $\{u, v\}$. Let $F=B+e$ and $H=G-(V(F)-\{u, v\})-E(F)$. Note that, if $|E(H)| \geq 2$, then $\{F, H\}$ is a 2-separation of $G$.

Since $G$ is 2-connected, $F$ and $F / e$ are both 2-connected. Lemma 2.2(b) now implies that $F$ is nearly 3 -connected. Since $e$ belongs to the outer face of $F$, we can apply Lemma 2.2(a) to $F$ to deduce that $F / e$ is also nearly 3 -connected. Let $w_{F}$ be the effective weight of $F$ in $(G, \mathbf{w})$.

Claim 3.9 $w_{F} \in W_{2}$.

Proof: We first suppose that $F-e$ is 2 -connected. Since $F / e$ is 2 -connected we may apply Claim 3.7 to $F-e$ to deduce that $w_{F-e} \in W_{1}$. Hence

$$
w_{F}=w_{F-e}\left\|w_{e} \in W_{1}\right\| W_{2} \subseteq W_{2}
$$

by Lemma 3.5(e).

Thus we may assume that $F-e$ is not 2-connected. Let $B_{1}$ and $B_{2}$ be the blocks of $F-e$ which contain $u$ and $v$ respectively. By Claim 3.7, we have $w_{B_{1}}, w_{B_{2}} \in W_{1}$. If $F-e=B_{1} \cup B_{2}$ this gives

$$
w_{F}=\left(w_{B_{1}} \bowtie_{q} w_{B_{2}}\right)\left\|w_{e} \in\left(W_{1} \bowtie_{q} W_{1}\right)\right\| W_{2} \subseteq W_{2}
$$

by Lemma 3.5(d). Thus we may suppose that $F-e \neq B_{1} \cup B_{2}$. The fact that $G$ is nearly 3 -connected now implies that $F-e$ has exactly three blocks $B_{1}, B_{2}, B_{3}$ and that $\left|E\left(B_{3}\right)\right|=1$. Then $w_{B_{3}}=-1$ and hence

$$
w_{F-e}=\left(w_{B_{1}} \bowtie_{q} w_{B_{3}}\right) \bowtie_{q} w_{B_{2}} \in\left(W_{1} \bowtie_{q}[-1]\right) \bowtie_{q} W_{1} \subseteq W_{1} \bowtie_{q} W_{1}
$$

by Lemma 3.4(c). Thus

$$
w_{F}=w_{F-e}\left\|w_{e} \in\left(W_{1} \bowtie_{q} W_{1}\right)\right\| W_{2} \subseteq W_{2}
$$

by Lemma $3.5(\mathrm{~d})$.

Let $\left(F+h,\left.\mathbf{w}\right|_{F},-1\right)$ be obtained from $\left(F,\left.\mathbf{w}\right|_{F}\right)$ by adding a new edge $h=u v$ to $F$ with weight -1 , and $\left(H+e_{F},\left.\mathbf{w}\right|_{H}, w_{F}\right)$ be obtained from $\left(H,\left.\mathbf{w}\right|_{H}\right)$ by adding an edge $e_{F}=u v$ with weight $w_{F}$. By Lemma 2.4,

$$
Z_{G}(q, w)=\frac{Z_{F+h}\left(q,\left.\mathbf{w}\right|_{F},-1\right)}{q(q-1)} Z_{H+e_{F}}\left(q,\left.\mathbf{w}\right|_{H}, w_{F}\right)
$$

By Lemma 2.5(a), $Z_{F+h}\left(q,\left.\mathbf{w}\right|_{F},-1\right)=Z_{F}\left(q,\left.\mathbf{w}\right|_{F-e},-1\right)$. Substituting into (16) we obtain

$$
Z_{G}(q, \mathbf{w})=\frac{Z_{F}\left(q,\left.\mathbf{w}\right|_{F-e},-1\right)}{q(q-1)} Z_{H+e_{F}}\left(q,\left.\mathbf{w}\right|_{H}, w_{F}\right) .
$$



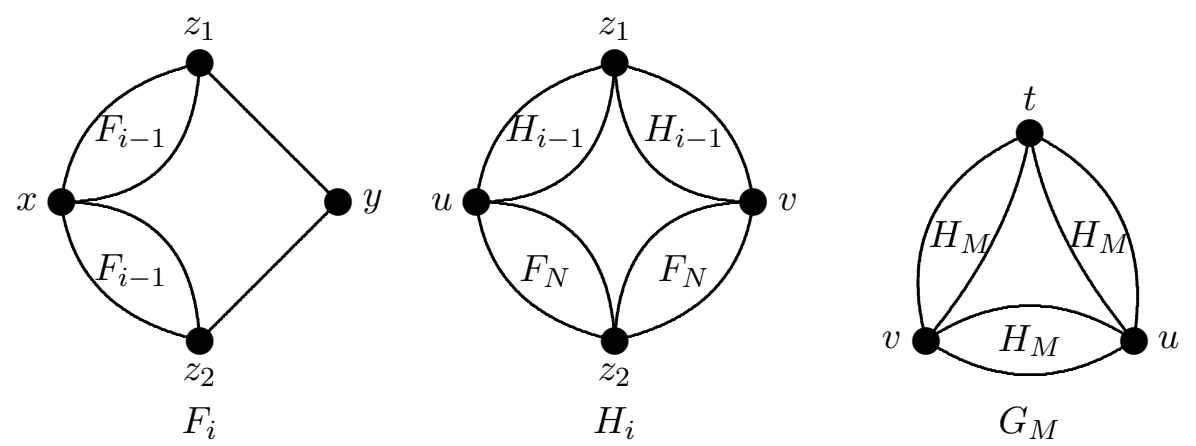

Figure 2: The graphs $F_{i}, H_{i}$ for $i \geq 2$ and the graph $G_{M}$.

Since $q>1$ and $|V(F)|+|V(H)|=n+2$, we may apply induction to $\left(F, q,\left.\mathbf{w}\right|_{F-e},-1\right)$ and $\left(H+e_{F}, q,\left.\mathbf{w}\right|_{H}, w_{F}\right)$ to deduce that $(-1)^{n} Z_{G}(q, \mathbf{w})>0$.

Theorem 1.1 follows immediately from Theorem 3.1.

\section{A recursive construction}

We use $K_{n}\left(v_{1}, v_{2}, \ldots, v_{n}\right)$ to denote a complete graph with vertices $v_{1}, v_{2}, \ldots, v_{n}$. We will construct an infinite family of nearly 3 -connected plane graphs with chromatic roots tending to $\gamma$. We use the diamond operation which replaces an edge $u v$ of a graph $G$ by a 4 -cycle $u z_{1} v z_{2} u$ where $z_{1}, z_{2} \notin V(G)$. As a first step we construct a sequence of 2-rooted plane graphs $F_{1}(x, y), F_{2}(x, y), \ldots$ with root vertices $x, y$ such that every 2 -vertex cut of $F_{i}(x, y)$ contains $x$, and such that the effective weights of the $F_{i}(x, y)$ tend to $b_{1}$ from below. We let $F_{1}=K_{2}(x, y)$. For $i \geq 2$, we construct $F_{i}$ from $F_{i-1}$ by performing the diamond operation on each edge incident to $x$, see Figure 2. Let $w_{i}$ be the effective weight of $F_{i}$ when all edge weights are equal to -1 .

Lemma 4.1 Suppose $q \in(1,1.25]$. Then:

(a) $-1 \leq w_{i}<b_{1}$ for all $i \geq 1$;

(b) $w_{i}>w_{i-1}$ for all $i \geq 2$;

(c) $\lim _{n \rightarrow \infty} w_{n}=b_{1}$.

Proof: (a) We have $w_{1}=-1<b_{1}$. Furthermore, for any $i \geq 2$,

$$
w_{i}=\left[w_{i-1} \bowtie_{q}(-1)\right] \|\left[w_{i-1} \bowtie_{q}(-1)\right] .
$$

Since for any $w \in \mathbb{R}$, we have $w \| w=(w+1)^{2}-1 \geq-1$, this immediately implies that $w_{i} \geq-1$. Furthermore, since $w_{1} \in W_{1}$ we may use (17), Lemma 3.4(b),(c) and induction to deduce that $w_{i} \in\left(W_{1} \bowtie_{q}[-1]\right) \|\left(W_{1} \bowtie_{q}[-1]\right) \subset W_{1}$. Hence $w_{i}<b_{1}$. 
(b) Let $f(w)=\left[w \bowtie_{q}(-1)\right] \|\left[w \bowtie_{q}(-1)\right]$. Then

$$
f(w)-w=-w\left(w^{2}+w(2 q-1)+q^{2}-1\right)(q-1+w)^{-2}
$$

Since $b_{1}$ is the smallest real root of $w^{2}+w(2 q-1)+q^{2}-1=0$ we have $f(w)-w>0$ for all $w<b_{1}$. We can now use (a) to deduce that $w_{i}-w_{i-1}=f\left(w_{i-1}\right)-w_{i-1}>0$.

(c) We have shown that $\left\{w_{i}\right\}$ is a strictly increasing sequence which is bounded above by $b_{1}$. This implies that $\left\{w_{i}\right\}$ must converge to a limit $\alpha$ which evidently must satisfy $\alpha \leq b_{1}$ and $f(\alpha)=\alpha$. Hence $\alpha=b_{1}$.

Let $\mathcal{F}$ denote the family of nearly 3 -connected plane graphs. For $G \in \mathcal{F}$ we use $Z_{G}(q, w,-1)$ to denote the multivariate Tutte polynomial for $G$ with all edge weights on the outer face equal to $w$ and all other edge weights equal to -1 .

Let $F(x, y)$ be a 2-rooted 2-connected plane graph with root vertices $x, y$ lying on the outer face of $F$, and with the property that all 2-vertex cuts of $F$ contain $x$. Let $w_{F}$ denote the effective weight of $F$ when all edges have weight -1 . We use $F$ to define a modified diamond operation on $\mathcal{F}$. Given $G \in \mathcal{F}$ with outer face $C$, the $F$-diamond operation replaces an edge $u v$ on $C$ by a graph $D=D_{1} \cup D_{2} \cup D_{3} \cup D_{4}$ where $V(D) \cap V(G)=\{u, v\}, D_{1}=K_{2}\left(u, z_{1}\right)$, $D_{2}=K_{2}\left(v, z_{1}\right), D_{3}=F\left(v, z_{2}\right), D_{4}=F\left(u, z_{2}\right)$, and $z_{1}$ lies on the outer face of the new graph. Let $\diamond_{F}(G)$ denote the graph obtained by applying the $F$-diamond operation to every edge of $C$. The fact that all 2-vertex cuts of $F$ contain $x$ implies that $\nabla_{F}(G) \in \mathcal{F}$. We may use Lemma 2.4 to express $Z_{\diamond_{F}(G)}(q, w,-1)$ in terms of $Z_{G}(q, \diamond(w),-1)$ where $\diamond(w)=\left(w \bowtie_{q} w\right) \|$ $\left(w_{F} \bowtie_{q} w_{F}\right)$. We first replace both copies of $F$ in each copy of $D$ in $\diamond_{F}(G)$ by edges of weight $w_{F}$, and then replace each resulting $C_{4}$ by an edge of weight $\diamond(w)$. Lemma 2.4 then gives

$$
Z_{\diamond_{F}(G)}(q, w,-1)=(q+2 w)^{m}\left(q+2 w_{F}\right)^{m}\left(\frac{Z_{F+h}(q,-1)}{q(q-1)}\right)^{2 m} Z_{G}(q, \diamond(w),-1)
$$

where $F+h$ is obtained from $F$ by adding a new edge $h=x y$, and $m=|E(C)|$.

Theorem 4.2 For each $q \in(\gamma, 1.25]$, there exits a nearly 3-connected plane graph $G$ such that $P_{G}\left(q_{1}\right)=0$ for some $q_{1} \in(\gamma, q]$.

Proof: Choose $q \in(\gamma, 1.25]$ and let

$$
h(w, b)=\left(b^{2}-2 b-q\right) w^{2}+\left(2 b^{2}-2 b q-q^{2}\right) w+b^{2} q .
$$

We have $2 b_{1}+q=-q+1-\sqrt{5-4 q}<0$ for all $q \in(1,1.25)$. The definition of $W_{1}$ and the fact that $q>\gamma$ now imply that $h\left(w, b_{1}\right)=g(w)>0$ for all $w \in \mathbb{R}$. Thus $\left(2 b_{1}^{2}-2 b_{1} q-q^{2}\right)^{2}-$ $4 b_{1}^{2}\left(b_{1}^{2}-2 b_{1}-q\right)<0$. By continuity, there exits $b_{0} \in\left(-1, b_{1}\right)$ such that $h(w, b)>0$ for all 
$w \in \mathbb{R}$ and all $b \in\left(b_{0}, b_{1}\right)$. Since $\left\{w_{i}\right\}$ is a strictly increasing sequence which converges to $b_{1}$ by Lemma 4.1, we may choose a positive integer $N=N(q)$ such that $w_{N} \in\left(b_{0}, b_{1}\right)$.

Recall that $w_{N}$ is the effective weight of the 2-rooted graph $F_{N}(x, y)$. We use the $F_{N}$-diamond operation to give a recursive construction for our required graph $G$. Put $G_{1}=K_{3}(t, u, v)$ and, for $i \geq 2$, let $G_{i}=\diamond_{F_{N}}\left(G_{i-1}\right)$. We will show that $P_{G_{i}}\left(q_{1}\right)=0$ for some $i \geq 1$ and some $q_{1} \in(\gamma, q]$.

Let $H_{1}(u, v)=K_{2}(u, v)$ and, for $i \geq 2$, let $H_{i}(u, v)$ be the 2-rooted subgraph of $G_{i}$ obtained from $H_{i-1}(u, v)$ by applying the $F_{N}$-diamond operation to all edges of $H_{i-1}(u, v)$ which lie on the outer face of $G_{i-1}$. For $i \geq 2$ we have $H_{i}=A \cup B \cup C \cup D$ where $A=H_{i-1}\left(u, z_{1}\right)$, $B=H_{i-1}\left(v, z_{1}\right), C=F_{N}\left(v, z_{2}\right)$ and $D=F_{N}\left(u, z_{2}\right)$, and $z_{1}$ lies on the outer face of $G_{i}$, see Figure 2. Let $r_{i}$ be the effective weight of the 2-rooted subgraph $H_{i}$ when all edges have weight -1 . We will show that $r_{M} \geq-q / 2$ for some $M \geq 1$.

Suppose to the contrary that $r_{i}<-q / 2$ for all $i \geq 1$. We have $r_{1}=-1$ and, since the effective weight of $F_{N}(x, y)$ is $w_{N}, r_{i+1}=\left[r_{i} \bowtie_{q} r_{i}\right] \|\left[w_{N} \bowtie_{q} w_{N}\right]$ for all $i \geq 1$. Hence

$$
r_{i+1}-r_{i}=\left[r_{i} \bowtie_{q} r_{i}\right] \|\left[w_{N} \bowtie_{q} w_{N}\right]-r_{i}=h\left(r_{i}, w_{N}\right)\left(q+2 r_{i}\right)^{-1}\left(q+2 w_{N}\right)^{-1}>0
$$

since $w_{N} \in\left(b_{0}, b_{1}\right)$. Thus $\left\{r_{i}\right\}$ is a strictly increasing sequence which is bounded above by $-q / 2$. This implies that $\left\{r_{i}\right\}$ must converge to a limit $\beta$ which evidently must satisfy $h\left(\beta, w_{N}\right)=0$. This is impossible since $h\left(w, w_{N}\right)>0$ for all $w \in \mathbb{R}$. Hence we may choose a positive integer $M$ such that $r_{M} \geq-q / 2$.

Consider the graph $G_{M}$. We have $G_{M}=R \cup S \cup T$ where $V(R) \cap V(S)=\{t\}, V(S) \cap V(T)=$ $\{u\}, V(T) \cap V(R)=\{v\}$, and each of $R, S, T$ is a 2-rooted subgraph isomorphic to $H_{M}(x, y)$,

see Figure 2. We may use (18) and the fact that $G_{i}$ has an even number of edges on its outer face for all $i \geq 2$ to deduce that

$$
P_{G_{M}}(q)=Z_{G_{M}}(q,-1)=(\text { nonnegative prefactor }) Z_{K_{3}}\left(q, r_{M}\right) \text {. }
$$

We have $Z_{K_{3}}\left(q, r_{M}\right)=q\left(r_{M}^{3}+3 r_{M}^{2}+3 q r_{M}+q^{2}\right)>0$ since $r_{M} \geq-q / 2$ and $q \in(1,2)$. Thus $P_{G_{M}}(q) \geq 0$. On the other hand $P_{G_{M}}(\gamma)<0$ by Theorem 1.1. Continuity now implies that $P_{G_{M}}\left(q_{1}\right)=0$ for some $q_{1} \in(\gamma, q]$.

\section{Open Problems}

It seems difficult to adapt the inductive proof technique used in this paper for the family of 3-connected graphs. There are, however, other families of 'nearly 3-connected graphs' for which a similar approach may work. 
Conjecture 5.1 Let $G$ be a 2-connected plane graph on $n$ vertices and let $C$ be the bounding cycle of the outer face of $G$. Suppose that, for all 2-separations $\left\{G_{1}, G_{2}\right\}$ of $G$, we have $V\left(G_{1}\right) \cap V\left(G_{2}\right) \subseteq V(C)$. Then $(-1)^{n} P_{G}(q)>0$ for all $q \in(1, \delta]$ where $\delta \sim 1.225 \ldots$ is a root of $x^{4}-4 x^{3}+4 x^{2}-4 x+4=0$.

Conjecture 5.2 Let $G$ be a 2-connected plane graph on $n$ vertices and let $C$ be the bounding cycle of the outer face of $G$. Suppose that, for all 2-separations $\left\{G_{1}, G_{2}\right\}$ of $G$, we have $E(C) \cap E\left(G_{1}\right) \neq \emptyset \neq E(C) \cap E\left(G_{2}\right)$. Then $(-1)^{n} P_{G}(q)>0$ for all $q \in(1, \rho]$ where $\rho \sim 1.430 \ldots$ is a root of $3 x^{10}-19 x^{9}+54 x^{8}-114 x^{7}+303 x^{6}-831 x^{5}+1704 x^{4}-2540 x^{3}+2400 x^{2}-1344 x+512=$ 0 .

Conjecture 5.3 Let $G$ be a 2-connected graph on $n$ vertices and $x$ be a vertex of $G$. Suppose that, for all 2-separations $\left\{G_{1}, G_{2}\right\}$ of $G$, we have $x \in V\left(G_{1}\right) \cap V\left(G_{2}\right)$. Then $(-1)^{n} P_{G}(q)>0$ for all $q \in(1,1.25]$.

The zero-free intervals given in Conjectures 5.1, 5.2 and 5.3 would be best possible. The family of graphs obtained recursively from $K_{3}$ by applying the diamond operation to each edge on the outer face satisfies the hypotheses of Conjecture 5.1 and has a chromatic root converging to $\delta$ from above. The family of graphs obtained recursively from $K_{3}$ by replacing each edge $u v$ on the outer face by the 2-rooted subgraph $F$ where $V(F)=\left\{u, v, x_{1}, x_{2}, x_{3}, x_{4}\right\}$ and $E(F)=\left\{u x_{1}, x_{1} x_{2}, x_{2} x_{3}, x_{3} v, x_{4} u, x_{4} x_{2}, x_{4} v\right\}$ satisfies the hypotheses of Conjecture 5.2 and has a chromatic root converging to $\rho$ from above. The family of graphs obtained recursively from $K_{3}(x, y, z)$ by applying the diamond operation to each edge incident to $x$ satisfies the hypotheses of Conjecture 5.3 and has a chromatic root converging to 1.25 from above.

\section{References}

[1] K. Appel and W. Haken, Every planar map is 4-colourable. Part I: Discharging, Illinois J. Math 21 (1977) 429-490.

[2] K. Appel, W. Haken and J. Koch, Every planar map is 4-colourable. Part II: Reducibility, Illinois J. Math 21 (1977) 491-567.

[3] G.D. Birkhoff, A determinant formula for the number of ways of colouring a map,Chromatic polynomials, Ann. of Math. 14 (1912), 42-46.

[4] G.D. Birkhoff and D.C. Lewis, Chromatic polynomials, Trans. Amer. Math. Soc. 60 (1946), 355-451.

[5] B. Jackson, A zero-free interval for chromatic polynomials of graphs, Combinatorics, Probability and Computing 2 (1993) 325-336. 
[6] B. Jackson, Zeros of Chromatic and Flow Polynomials of Graphs, J. Geom., 76 (2003) 95-109.

[7] B. Jackson and A. D. Sokal) Zero-free Regions for Multivariate Tutte Polynomials (alias Potts-model Partition Functions) of Graphs and Matroids, J. Combinatorial Theory(B), 99 (2009) 869-903

[8] W. Mader, Ecken vom Grad $\mathrm{n}$ in minimalen n-fach zusammenhängenden Graphen, Archive der Mathematik 23 (1972) 219-224.

[9] N. Robertson, D. Sanders, P. Seymour and R. Thomas, The four colour theorem, J. Combinatorial Theory, Series B 70 (1997) 2-44.

[10] G. F. Royle, Recent results on chromatic and flow roots of graphs and matroids, in Surveys in Combinatorics 2009, S. Huczynska, J. D. Mitchell, C. M. Roney-Dougal eds. CUP Cambridge 2009,

[11] A. D. Sokal, The multivariate Tutte polynomial (alias Potts model) for graphs and matroids, in Surveys in Combinatorics 2005, B. Webb ed. CUP Cambridge 2005, 173226.

[12] C. Thomassen, The zero-free intervals for chromatic polynomials of graphs, Combinatorics, Probability and Computing 6 (1997) 497-506.

[13] W.T. Tutte, Chromials, Springer Lecture Notes in Mathematics 411 (1974), 243-266.

[14] H. Whitney, A logical expansion in mathematics, Bull Amer. Math. Soc. 38 (1932) 572579.

[15] H. Whitney, The coloring of graphs, Ann. of Math. 33 (1932) 688-718. 